\title{
LOS VESREÍSMOS COMO BASES LÉXICAS
}

\author{
Piotr Sorbet \\ Universidad de Maria Curie-Skłodowska \\ http://dx.doi.org/10.18778/8220-201-4.29
}

\section{Resumen}

Como es comúnmente sabido, el vesre constituye uno de los numerosos contrastes entre el español europeo y las variantes hispanoamericanas. En efecto, es un mecanismo de creación léxica cuya productividad en la España contemporánea es prácticamente nula, mientras que en los diversos países hispanoamericanos es un procedimiento de formación de palabras relativamente recurrente. Sin embargo, su análisis detallado no debe consistir solo en la descripción de los vesreísmos, sino que también requiere, según nuestra perspectiva, el estudio de las unidades léxicas que se han creado a base de ellos. De ahí que, en esta ocasión, pretendamos ocuparnos de las palabras creadas en el marco del vesre y tratarlas como bases léxicas para la formación de otros vocablos. Para este fin, vamos a basarnos, sobre todo, en una serie de diccionarios, lo que significa que nuestro trabajo va a tener un carácter lexicográfico.

Palabras clave: Vesre, vesreísmo, palabras devesreicas, derivación devesreica, truncación devesreica.

\section{1. \\ Introducción}

El motivo que nos ha incitado a escribir este trabajo radica en nuestra convicción según la cual cualquier unidad léxica de una lengua tiene la posibilidad de diferenciar las variantes de un idioma de dos maneras principales. 
En primer lugar, el simple hecho de que alguna palabra exista en una variante y no en otra provoca que entre estas variantes se produzca un contraste dialectal. Así, en español en las zonas andinas, por razones étnicas, hay más quechuismos que en México donde, por su parte, los aportes nahuas son mayores que en Perú. En la región bonaerense, debido a los procesos inmigratorios de finales del siglo XIX y principios del siglo XX, se registran más italianismos que en la zona caribeña. De esta manera numerosos quechuismos, nahuatlismos e italianismos causan que las distintas variantes hispanoamericanas, por un lado, se diferencien entre sí, y, por otro lado, se opongan a las variedades que se utilizan en Europa.

En segundo lugar, una palabra, cuyo uso está restringido diatópicamente, puede llegar a ser una palabra primitiva que después sirve para la acuñación de otras unidades lingüísticas. Estas aumentan aún más los contrastes entre las distintas variantes de una lengua y, por esta razón, constituyen un componente medular en el proceso de la diversificación diatópica del idioma en cuestión.

Ahora bien, a pesar de que en español los múltiples mecanismos de creación léxica (derivación, composición, etc.), según las regiones, presentan ciertas diferencias, todos estos recursos lexicogénicos se emplean semejantemente en todas las partes. Así, por ejemplo, el sufijo -ero forma nombres de oficios relacionados con la cosa expresada por el nombre primitivo y se utiliza en todas las variantes del español. Esto no impide que existan derivados que se utilicen solo en ciertas zonas: Bo autero $(\leftarrow$ auto) 'ladrón que se dedica a robar vehículos, accesorios u objetos que se hallan en el interior de aquellos' (Bo-DCo, DASALE), Ar, Ur colectivero $(\leftarrow$ colectivo) 'conductor de un colectivo' (DEAr, DASALE), etc. Los verbos romper, lavar sirven para crear diversos compuestos verbonominales (rompecabezas, rompeolas, lavaplatos, lavavajillas). Sin embargo, las formas rompe y lava se usan igualmente para acuñar numerosas palabras que se registran solo en ciertas zonas del mundo hispanohablante: [Hispam] rompenueces 'cascanueces' (DUE, DASALE, DLE), Ar, Ur rompevientos 'anorak' (DASALE, DUE, DLE, DEAr), Ch limpiapiés 'felpudo' (DASALE, 
$D E U C h)^{1}$. En otros casos la diferencia dialectal radica en la frecuencia de uso de algún vocablo (rompehuelgas) o en que una palabra tiene distintos significados según las regiones (lavaplatos) (cfr. DUE). A pesar de la existencia de estas voces, la derivación mediante el sufijo -ero y la composición basada en los verbos romper y limpiar funcionan de manera parecida en todo el mundo hispánico ${ }^{2}$.

No obstante, el español tiene un mecanismo de creación léxica que no ha recibido, desde nuestra perspectiva, la atención debidamente merecida. Se trata del vesre cuya productividad en ciertas zonas es nula o escasa, pero en otras es más recurrente de lo que puede parecer a primera vista. Por lo tanto, su análisis debería consistir, por una parte, en la descripción de los vesreísmos y, por otra, en el estudio de las unidades que han sido creadas posteriormente a base de ellos. En efecto, estamos convencidos de que tanto los vesreísmos como los elementos devesreicos contribuyen al proceso de la diferenciación dialectal del español.

Queremos hacer hincapié en que, a pesar de que el vesre es sobre todo un fenómeno oral, las unidades léxicas que vamos a tratar se registran, en la mayoría de los casos, en diversos diccionarios, lo que significa que este trabajo tiene un carácter lexicográfico. La lista de las obras consultadas se encuentra junto con las siglas empleadas en la bibliografía final. Notemos que en esta ocasión vamos a centrarnos privilegiadamente en el vesre argentino, aunque vamos a suministrar igualmente algunos ejemplos del vesre boliviano, chileno y peruano. Los vesres de las demás variantes del español constituirán el objeto de otros trabajos.

Asimismo, es necesario recalcar que en esta investigación no vamos a hacer la distinción entre los vesreísmos o vocablos devesreicos que se utilizan contemporáneamente de los que, aparentemente, ya han caído en desuso.

Dado que no queremos repetir las descripciones que ya hemos realizado en otras ocasiones para la adecuada comprensión

1 Las marcas diatópicas presentadas aquí son muy aproximativas.

2 Sobre el estatus de este tipo de compuestos, muy productivos en las lenguas románicas, $c f r$. Val Álvaro (1999: 4788-4789). 
de este fenómeno lingüístico, remitimos a nuestros trabajos anteriores (Sorbet, 2014, 2016a, 2016b, 2017, 2018). A continuación vamos a presentar la riqueza de las formas devesreicas, dividiéndolas según los recursos de la creación léxica de los que dispone el español. En esta ocasión nos limitamos a la derivación y al truncamiento.

\section{2. \\ Derivación}

Sin sombra de duda, el procedimiento más productivo de acuñación de vocablos nuevos en español es la derivación. Esta se realiza por medio de tres procesos diferentes: la prefijación, la sufijación y la parasíntesis (NGDLE, 2010: 7) ${ }^{3}$. Los afijos, en la primera de ellas se anteponen a la raíz, en la segunda se posponen y en la última se colocan simultáneamente antes y después de la base léxica. Tengamos en cuenta que en español existen diversos afijos cuyo uso se limita prácticamente solo a unas zonas de habla hispana, por ejemplo, ciertos sufijos tanto potestativos como aspectuales (Bajo Pérez, 1997: 48-53). Hay también otros que se utilizan en todas las partes de habla hispana, por ejemplo, -ero (sufijo que forma nombres de oficio relacionado con la cosa expresada por el nombre primitivo) o -ista, pero se añaden a ciertas raíces solo en algunas zonas: auto $\rightarrow$ Bo autero (cfr. supra) (Bo-DCo). Finalmente, hay un grupo muy numeroso de derivados que se crean agregando algún afijo a una base léxica cuyo uso presenta restricciones diatópicas, diastráticas, diafásicas, diamésicas, etc. Este es el caso de los derivados devesreicos. Además, no en pocas ocasiones los vesreísmos sirven como bases para la formación de

3 Hay que tener presente que hasta hace relativamente poco, la prefijación se había ubicado en la composición ( $c f r$. Gómez Torrego 2002 [1997]: 18). Además, ya que en la parasíntesis se hace uso simultáneo de la prefijación y la sufijación, la parasíntesis participa de la derivación y la composición (NGDLE, 2010: 7-8). 
familias léxicas. Dado que sus raíces constituyen palabras limitadas diatópicamente, es posible sostener que las familias léxicas, que estos vocablos crean, deben tener también las mismas restricciones. Notemos que los vesreísmos se acuñan a base de vocablos que a menudo no pertenecen, por muy controvertido que sea el término, al habla estándar (argot, jerga, etc.). De ahí que los derivados devesreicos igualmente se sitúen a menudo al margen o incluso fuera de ella.

Deseamos recalcar que es difícil exponer una lista completa de todos los derivados devesreicos. Por esta razón, para que la lista no sea demasiado extensa, a continuación vamos a presentar solo una selección de afijos y cada uno de ellos va a estar acompañado solo de un ejemplo de derivado. En la lista daremos a conocer igualmente una breve definición del afijo basada, ante todo, en el $D U E$, así como al menos una de las acepciones del derivado que extraemos de nuestro corpus lexicográfico:

-aje Sufijo colectivo que forma nombres derivados de otros nombres o de verbos.

hembra $\rightarrow$ brame 'Concubina, manceba' (NDLJG, DELOC) $\rightarrow$ bramaje 'conjunto o grupo de mujeres' (DELOC, NDLJG) ${ }^{4}$; -ada Sufijo despectivo.

botón $\rightarrow$ tombo 'Miembro del cuerpo de la policía' (DiPerú, $D E P P e, B O-D C O, D E L O C, D A S A L E) \rightarrow$ tombeada 'Conjunto de policías' (Bo-DCo);

-ador Sufijo que forma nombres de agente.

pagar $\rightarrow$ garpar 'Pagar una persona sus deudas' (DEAr, DE$L O C, B O-D C O) \rightarrow$ garpador 'Persona que siempre paga sus deudas puntualmente' (DEAr, DELOC);

-ar Sufijo del infinitivo de los verbos de la primera conjugación derivados de nombres.

4 Según el DELOC y el NDLJG bramaje es un vesreísmo irregular de hembraje. Sin embargo, la forma de la primera sílaba (bra-) sugiere que puede tratarse en este caso más bien de un derivado creado a base del vesreísmo brame que se registra en los dos diccionarios que hemos consultado. 
batidor $\rightarrow$ ortiba 'Malhumorado, cortado; que rechaza invitaciones y propuestas para divertirse' (DELOC, DEAr) $\rightarrow$ ortibarse 'Enojarse' (DELOC);

-ato/a Sufijo que en adjetivos indica una cualidad.

viejo $\rightarrow$ jovie 'Hombre de edad avanzada' (DEAr, DELOC, ND$L J G) \rightarrow$ jovato 'Persona vieja o de edad avanzada' (DEAr, DELOC, NDLJG, DUE, Bo-DCo) ${ }^{5}$;

-e Sufijo para formar nombres de acción y efecto derivados de verbos.

pagar $\rightarrow$ garpar 'Pagar una persona una determinada suma de dinero' (DELOC, DEAr, Bo-DCo) $\rightarrow$ garpe 'pago' (DELOC);

-ear Sufijo del infinitivo de los verbos de la primera conjugación derivados de nombres.

garcha $\rightarrow$ chagar 'Pene' (DELOC, NDLJG) $\rightarrow$ chagarear 'Copular' (DELOC, NDLJG);

-ería Sufijo de abundancia, cualidad, conjunto.

botón $\rightarrow$ tombo 'Policía generalmente uniformado' (LPP, DEPPe, MPFP, DELOC, NDLJG, Bo-DCo, DASALE, DiPerú) $\rightarrow$ tombería 'Grupo de policías uniformados' (LPP, DEPPe, MPFP, DiPerú);

-ero Sufijo de nombres de utensilios.

papel $\rightarrow$ pelpa 'Billete de banco' (LDin) $\rightarrow$ pelpera 'Billetera' (DELOC, LDin);

-il Sufijo despectivo que forma adjetivos con el significado de 'propio de' o 'con aspecto de'.

botón $\rightarrow$ tombo 'Agente de policía' (DELOC, LPP, DEPPe, DiPerú, MPFP, NDLJG, Bo-DCo, DASALE) $\rightarrow$ tomberil 'Relativo a la policía' (DEPPe);

-illo Sufijo diminutivo.

botón $\rightarrow$ tombo 'Agente de policía' ( $L P P, D E P P e, M P F P, D E-$ LOC, NDLJG, Bo-DCo, DASALE) $\rightarrow$ tombillo 'Miembro de la policía encargado de resguardar el orden público en las calles' (Bo-DCo);

5 Sin sombra de duda, la voz jovato es una forma analógica al vocablo novato (DUE, NDLJG, DELOC). 
-ista Sufijo que forma nombres de agente, oficio o profesión.

farra $\rightarrow$ rafa 'Diversión' (DELOC) $\rightarrow$ rafista 'Juerguista, aficionado a la juerga o farra' (DELOC);

-ito/a Sufijo diminutivo.

mujer $\rightarrow$ germa 'Mujer adulta' $\rightarrow$ germita 1 . 'Hombre homosexual'; 2. 'Mujer joven' (Bo-DCo, DJPe);

-ón Sufijo aumentativo.

batidor $\rightarrow$ ortiba 'Persona que revela a una autoridad, generalmente a la policía, quién es el autor de un acto delictivo o censurable, o que da información importante sobre el mismo' (DEAr, DELOC, DASALE) $\rightarrow$ ortibón 'Forma aumentativa de ortiba' (DELOC).

Asimismo, aparte de los afijos que hemos enumerado supra, es necesario destacar que a las formas vésricas a veces se añaden elementos propios de ciertos sociolectos, jergas, argot, etc. De ahí que no sea sorprendente que en el lunfardo y en el cocoliche (Mezo-Zilio, 1960: 90) se utilice el sufijo de tono italianizante -eli (de origen italiano en el que se lo utiliza para la formación de diminutivos y de muchos apellidos que se agrega a numerosas voces): loco $\rightarrow$ locateli, duro $\rightarrow$ dureli, zurdo $\rightarrow$ zurdeli. Podemos encontrarlo en la voz jovateli 'Persona vieja o de edad avanzada' que se compone del elemento -eli y del vesreísmo jovie $\leftarrow$ viejo $(D E A r)$.

Es importante mencionar también que los derivados devesreicos se convierten en ciertas ocasiones en bases para la formación de otros vocablos que se forjan mediante los afijos o truncamientos (cfr. infra) originando, por consiguiente, una cadena lexicogénica. Un ejemplo de ella nos gustaría presentar a continuación.

El vocablo bacán 'persona que vive sin privaciones, gozando de los placeres y del lujo de su posición acomodada' (DEAr, NDLJG, $D E L O C$ ) se vesrea en camba 'dueño de una mujer' (DEAr, ND$L J G, L D i n)$ y a este sustantivo se añade, primeramente, el sufijo afectivo - usa para crear la palabra cambusa (DELOC, $N D L J G) \mathrm{y}$, segundamente, a esta se agrega el 'sufijo de abundancia, cualidad, 
conjunto' -ría dando origen a cambusería 'condición de bacán' (DELOC, NDLJG).

Aunque existen otros casos de cadenas lexicogénicas de este tipo, debido a falta de espacio, no podemos permitirnos mostrarlas aquí. No obstante, ya es posible afirmar que hemos demostrado la existencia de los derivados dedevesreicos, es decir, palabras derivadas, formadas a base de derivados cuyo origen es un vesreísmo. Su proceso de formación se presenta esquemáticamente de la manera siguiente:

voz vesreable $\rightarrow$ vesreísmo + sufijo $\rightarrow$ derivado devesreico + sufijo $\rightarrow$ derivado dedevesreico (vesreamiento) (derivación sufijal) (derivación sufijal)

En relación con la productividad de los derivados devesreicos, es necesario notar que esta no en todos los casos es igual. De hecho, hay ciertos vesreísmos que son más productivos que otros. Algunos, aparentemente, no se transforman en bases léxicas, en cambio, otros constituyen el punto de partida para forjar uno, dos y más vocablos. Asimismo, en ciertas ocasiones, ya se ha llegado a crear familias léxicas devesreicas. Entre las más numerosas indiquemos:

a) tombo ( $\leftarrow$ botón): tomba (BDCo), tombeada (Bo-DCo), tombera (Bo-DCo), tombola (DJC), tombería (LPP, DEPPe, MPFP), tomberil (DEPPe), tombillo (Bo-DCo);

b) garcar ( $\leftarrow$ cagar): garca (DELOC, DEPPe), garcada (DELOC), garcador (DELOC), garcaino (DELOC), garco (DELOC) 6 .

Finalmente, es menester observar que la derivación a base de los vesreísmos se realiza exclusivamente mediante la sufijación. En efecto, de momento no hemos encontrado ningún ejemplo ni de prefijación ni de parasíntesis devesreica.

6 Fijémonos en que a estas palabras todavía hay que añadir sus diferentes variantes formales, así como las unidades fraseológicas en las que aparecen los vesreísmos. 


\section{Truncamiento}

Uno de los procedimientos más típicos del enriquecimiento del vocabulario es su tendencia a acortar las palabras. El truncamiento o abreviamiento (Alvar Ezquerra, 2006: 45) es el más común y se registra en todas las zonas hispanohablantes. Consiste en que una unidad léxica reduce su significante pero, en la mayoría de las veces, mantiene su significado y la clase de palabra. Sin embargo, se diferencia de la voz de partida no solo en la longitud, sino que también en el estilo al que pertenece (Casado Velarde, 1999: 5077). En efecto, el acortamiento es característico de la lengua (NGDLE, 2010: 10) o habla coloquial (Alvar Ezquerra, 2006: 45). Por tanto, es posible afirmar que el término acortado suele pertenecer a la variedad lingüística informal (Casado Velarde, 1999: 5077). Fijémonos, no obstante, en que entre las diferentes zonas de habla hispana existen numerosas discrepancias al respecto. En primer lugar, una forma acortada puede venir de dos palabras según las zonas: colegio $\rightarrow$ cole (DUE), Ar colectivo $\rightarrow$ cole $(D E L O C)$, Ch coletero $\rightarrow$ cole $(D A S A L E)$. En segundo lugar, una voz puede acortarse de diferentes maneras según las regiones: bicicleta $\rightarrow$ bici (DLE, DUE), CR, Pe bicla (DJPe, DEPPe: s.v. bicicletear; DASALE), Pe cleta (DEPPe: s.v. bicicletear). En tercer lugar, algunos términos acortados vienen de palabras que no se utilizan en todos los países de habla hispana, contribuyendo de esta manera a la diferenciación dialectal de una manera doble, primero, por la existencia del término de partida y, segundo, por la existencia del término acortado. Dado que, como hemos afirmado supra, los acortamientos suelen formar parte, por lo menos en la primera fase (Casado Velarde, 1999: 5077), de las variedades informales en las que suelen incluirse: jergas, argots, etc., no es sorprendente que en estos haya numerosos acortamientos. En efecto, los abreviamientos son muy comunes en el lunfardo, la replana, la jeringa, el coba, etc. De ahí que sea previsible que haya muchos vesreísmos que tengan formas truncadas. Entre ellas la mayor parte tiene su origen en las formas trisilábicas: garcar $(\leftarrow$ cagar) $(D E L O C, N D L J G) \rightarrow$ garcador $(D E-$ $L O C, N D L J G) \rightarrow$ garca 'homosexual' (DEPPe, DELOC, NDLJG); 
Pe camisa $\rightarrow$ samica $\rightarrow$ mica 'camisa' (DEPPe, DJPe, Bo-DCo); lunf. muñeca 'habilidad, capacidad para lograr un propósito' (NDLJG, $D E L O C) \rightarrow$ cañemu $(D E L O C) \rightarrow$ ñemu $(D E L O C, N D L J G) ;$ pabellón $\rightarrow$ bellompa 'pabellón de presos' (DELOC, NDLJG) $\rightarrow$ yompa (NDLJG, DELOC); pantalón $\rightarrow$ lompa (DELOC, NDLJG, DASALE, $D J P e, D E P P e, B o-D C o$ ).

Sin embargo, también existen vesreísmos que vienen de las palabras compuestas de cuatro sílabas. Estas tras el vesreamiento y el truncamiento dan origen a formas: di-: calzoncillos $\rightarrow$ zolcinllonca $(s)(D E L O C) \rightarrow$ zolcán / zolcan (DEPPe, DiPerú, LPP) y trisilábicas: uruguayo $\rightarrow$ yorugua $(D E L O C, D E P P e) \rightarrow$ yoruga (DELOC), lunf. loco $\rightarrow$ colo $(D E L O C) \rightarrow$ colifato $(D E L O C) \rightarrow$ coli$f a(D E L O C)$. Además, en ciertas ocasiones los vocablos, aunque experimentan el truncamiento, no reducen su número de sílabas: lunf. cafishio 'proxeneta' (DELOC, NDLJG) $\rightarrow$ shiofica (DELOC, $N D L J G) \rightarrow$ shofica (DELOC, NDLJG); lunf. mishio 'pobre, indigente' (DELOC, NDLJG) $\rightarrow$ shiome $(N D L J G) \rightarrow$ shome $(N D L J G)$. A pesar de ello, es plausible sostener que, sin contar ciertos casos excepcionales (colifa, yoruga), las palabras creadas por el truncamiento de vesreísmos están formadas, en la mayoría de las veces, de dos sílabas?.

El abreviamiento de los vesreísmos, como hemos visto en los ejemplos enumerados más arriba, se realiza a través de tres procedimientos básicos: la: aféresis (mica, ñemu, yompa), la apócope (colifa) y la síncope (shofica, shome). Asimismo, cabe destacar que la clase de palabras que más tiende a crear los acortamientos a base de vesreísmos es la de los sustantivos. Estos tras el vesreamiento guardan la clase de palabras de la que proceden, así como su género gramatical, por ejemplo, pabellón $(\mathrm{m})=$. yompa $(\mathrm{m}$.$) ,$ pantalón $(\mathrm{m})=.\operatorname{lompa}(\mathrm{m}$.$) , etc.$

7 Es necesario observar que en español el acortamiento en general suele llevar a la acuñación de vocablos disilábicos (Casado Velarde, 1999: 5079). 


\section{4.}

\section{Conclusiones y perspectivas para futuros trabajos}

El presente estudio demuestra que el fenómeno del vesre hispánico es más complejo de lo que puede parecer a primera vista. En efecto, resulta que no solo los vesreísmos fomentan la diversificación diatópica del español sino también las palabras devesreicas. Es necesario observar, no obstante, que existen numerosos otros vocablos devesreicos que se han forjado mediante otros procedimientos lexicogénicos (el floreo verbal, la composición, etc.) que aquí no hemos podido tratar.

Como hemos indicado al principio de esta contribución, nuestro análisis ha tenido un carácter meramente lexicográfico en el que, ante todo, hemos constatado la existencia de ciertas formas en los diccionarios. Sin embargo, creemos que sería digno de interés realizar otras investigaciones apoyadas en otros tipos de fuentes, por ejemplo: la prensa, las obras literarias, las letras de canciones, las páginas web, ya que en todas ellas es posible encontrar no solo vesreísmos, sino también voces creadas a base de ellas. Además, un trabajo basado en estas fuentes podría permitir formular ciertas observaciones más pormenorizadas de índole morfológica, semántica, pragmática, etc.

Finalmente, queremos insistir en que somos conscientes de que los vesreísmos y los vocablos devesreicos presentan numerosas diferencias diatópicas y estas deberían constituir uno de los temas de las futuras investigaciones que pronto deseamos realizar.

\section{Referencias bibliográficas}

Alvar Ezquerra, M. (2006), La formación de palabras en español, 6. ${ }^{a}$ ed., Arco/Libros: Madrid.

Bajo Pérez, E. (1997), La derivación nominal en español, Arco/ Libros: Madrid.

Casado Velarde, M. (1999), “Otros procesos morfológicos: acortamientos, formación de siglas y acrónimos”, en I. Bosque y V. Demonte (coords.), Gramática descriptiva de la lengua española, 
Vol. 3: Entre la oración y el discurso. Morfología, Madrid: Espasa Calpe, 5075-5096.

Gómez Torrego, L. (2002 [1997]), Gramática didáctica del español, SM: Madrid.

Meo-Zilio, G. (1960), "El 'cocoliche' rioplatense”, Boletín de filología, XVI, 61-119.

NGDLE (2010), Nueva gramática de la lengua española, Real Academia Española / Asociación de Academias de la Lengua Española, Madrid: Espasa.

Sorbet, P. (2014), “Análisis lingüístico del vesre porteño", Roczniki Humanistyczne, LXII, 123-134.

Sorbet, P. (2016a), "Aproximación a la descripción lingüística del vesre porteño", en J. Wilk-Racięska, A. Szyndler y C. Tatoj (eds.), Relecturas y nuevos horizontes en los estudios hispánicos, Vol. 4: Lingüística y didáctica de la lengua española, Katowice: Wydawnictwo Uniwersytetu Śląskiego, 173-183.

Sorbet, P. (2016b), "En torno al tratamiento lexicográfico de los vesreísmos”, Itinerarios, 23, 141-153.

Sorbet, P. (2017), “El vesre: un mecanismo de creación léxica”, Studia iberystyczne, 16, 151-167.

Sorbet, P. (2018): "Las convergencias y divergencias entre el vesre y el verlan” en J. Bień, B. Brzozowska-Zburzyńska, A. M. López González y W. Nowikow (eds.), Lingüistica hispánica en Polonia: tendencias y direcciones de investigación, serie: Manufactura Hispánica Lodziense, Vol. 4, Łódź-Lublin: Wydawnictwo Uniwersytetu Łódzkiego, 259-271.

Val Álvaro, J. F. (1999), “La composición”, en I. Bosque y V. Demonte (coords.): Gramática descriptiva de la lengua española, Vol. 3: Entre la oración y el discurso. Morfología, Madrid: Espasa, $4757-4842$.

\section{Diccionarios}

(DUECh) Academia Chilena de la Lengua (2010), Diccionario de uso del español de Chile, Santiago de Chile: MN Editorial Ltda.

(DASALE) Asociación de Academias de la Lengua Española (2010), Diccionario de americanismos, Lima: Santillana. 
(DEPPe) Calvo Pérez, J. (2014), Diccionario etimológico de palabras del Perú, Lima: Universidad Ricardo Palma.

(DiPerú) Calvo Pérez, J. (dir.) (2016), DiPerú. Diccionario de peruanismos, Lima: Compañía de Minas Buenaventura / Academia Peruana de la Lengua.

(Bo-DCo) Coello Vila, C. (1998), Diccionario coba. Sociolecto de la delincuencia boliviana, La Paz: Instituto Boliviano de Lexicografía y Otros Estudios Lingüísticos.

(DELOC) Conde, Ó. (2004), Diccionario etimológico del lunfardo, Buenos Aires: Taurus.

(LDin) Dellapiane Cálcena, C. (2007), Léxico del dinero, Buenos Aires: Academia Argentina de Letras.

(NDLJG) Gobello, J., Oliveri, M. (2010), Novísimo diccionario lunfardo, Buenos Aires: Corregidor.

(DEAr) Haensch, G., Werner, R. (directores) (2000), Diccionario del español de Argentina. Español de Argentina / Español de España, Madrid: Gredos.

(MPFP) Hildebrandt, M. (2011), Mil palabras y frases peruanas, Lima: Planeta Perú.

(DJPe) Larco Degregori, F. (2000), Diccionario de Jeringa Peruana, Lima: Cecosami.

(DUE) Moliner, M. (2008), Diccionario de uso del español, Madrid: Gredos, [CD-ROM].

(LPP) Portilla Durand, L. (2011), Léxico popular peruano, Lima: Universidad Ricardo Palma.

(DLE) Real Academia Española (2014), Diccionario de la lengua española, [en línea] <http://www.rae.es $>$ [fecha de consulta: 15.08.2018]. 